\title{
Autonomic nervous system and a 'vascular phase' in Takotsubo syndrome pathogenesis
}

John E. Madias

I very much enjoyed reading the Review by Akashi et al. (Epidemiology and pathophysiology of Takotsubo syndrome. Nat. Rev. Cardiol. doi:10.1038/nrcardio.2015.39), ${ }^{1}$ particularly the section on pathophysiology, which I have scrutinized. I share the views of the authors that "several pathophysiological explanations have been proposed for this syndrome and its intriguing appearance, and awareness is growing that these explanations might not be mutually exclusive" and, in this spirit, I have some remarks for their consideration. The excess of blood-borne catecholamines in Takotsubo syndrome points to direct cardiotoxic effects (catecholamineinduced myocardial injury) $;^{2}$ however, the epinephrine-mediated switch from the stimulatory $G_{a s}$ pathway to the cardioinhibitory $G_{\text {ai }}$ pathway in cardiomyocytes, ${ }^{3,4}$ and the calcium-dependent depression in contractility, point to an alteration in function-two intuitively distinct mechanisms for Takotsubo syndrome. One could envisage both mechanisms acting in tandem, or the last two preceding the first. The authors state that "increased extracellular fibrosis", along with myocardial oedema, are "generally associated with disturbed microcirculation", which ultimately "result in a mismatch between oxygen supply and demand in cardiomyocytes". ${ }^{1}$ However, one wonders whether the microcirculation is an epiphenomenon rather than the cause of Takotsubo syndrome.

The authors favour a primary pathogenetic role of blood-borne catecholamines in Takotsubo syndrome ${ }^{1}$ - something currently under scrutiny ${ }^{5}$ - and do not consider the possibility of an initiating pathogenetic influence from an autonomic adrenergic storm per se causing Takotsubo syndrome (as discussed previously ${ }^{6}$ ), with the surge of blood-borne catecholamines constituting an overspill. Connected to such a pathogenetic scenario is the notion that patients with underlying autonomic nervous system neuropathies (such as in diabetes mellitus) might be protected from developing Takotsubo syndrome. ${ }^{7}$ The 'synthetic' approach that the authors adopt in speculating about the pathogenesis of Takotsubo syndrome on page 6 of their article is convincingly plausible. The authors of a review about 'scorpion cardiomyopathy' which is indistinguishable from Takotsubo syndrome-refer to an early "vascular phase" preceding the "myocardial phase". This vascular phase, which conceivably occurs in the hyperacute phase of Takotsubo syndrome, might currently be overlooked, and so should be proactively sought after using early and frequent implementation of echocardiography. ${ }^{9,10}$

Inducible vasospasm with acetylcholine provocation of the conduit (epicardial) and/ or resistance (microcirculation) components of the coronary vasculature at follow-up might constitute a transient residue of the subacute phase of the illness-an epiphenomenon rather than the cause of Takotsubo syndrome. Akashi et al. state that the "density of $\beta$-adrenoceptors is highest in the apical myocardium of the left ventricle", which supports the emergence of apical akinesis/ dyskinesis according to "the $\beta 2$-adrenoceptor hypothesis". Conversely, "the higher sympathetic nerve density in the basal myocardium than in the apex", ${ }^{1}$ could be the mechanism for basal hyperkinesis mediated by the autonomic nervous system and a local myocardial surge in norepinephrine, ${ }^{6}$ leading secondarily to the Takotsubo syndrome apical akinesis/ dyskinesis. ${ }^{11}$ What is viewed as 'chemical myocarditis', 'toxic catecholamine injury', 'inflammation', or 'microcirculatory impairment' in Takotsubo syndrome, could conceivably be a consequence of the 'mechanical beating' imparted to the mid-ventricular and/ or apical myocardium by the hypercontractile base, ${ }^{11}$ manifesting during the difficult-toexplore hyperacute phase of the illness. Given the observation in patients with Takotsubo syndrome of migration of abnormalities in myocardial wall contraction during the same episode, the emergence of various topographical phenotypes of the disease (such as inverse Takotsubo syndrome) is not incongruent with the notion of early basal hyperkinesis at the inception of Takotsubo syndrome. ${ }^{12}$
Icahn School of Medicine at Mount Sinai, 1468 Madison Avenue, New York, NY 10029, USA.

madiasj@nychhc.org

Competing interests

The author declares no competing interests.

1. Akashi Y. J., Nef, H. M. \& Lyon, A. R. Epidemiology and pathophysiology of Takotsubo syndrome. Nat. Rev. Cardiol. http:// dx.doi.org/10.1038/nrcardio.2015.39.

2. Wittstein, I. S. et al. Neurohumoral features of myocardial stunning due to sudden emotional stress. N. Engl. J. Med. 352, 539-548 (2005).

3. Lyon, A. R., Rees, P. S., Prasad, S., Poole-Wilson, P. A. \& Harding, S. E. Stress (Takotsubo) cardiomyopathy-a novel pathophysiological hypothesis to explain catecholamine-induced acute myocardial stunning. Nat. Clin. Pract. Cardiovasc. Med. 5, 22-29 (2008)

4. Paur, H. et al. High levels of circulating epinephrine trigger apical cardiodepression in a $\beta_{2}$-adrenergic receptor/G-dependent manner: a new model of Takotsubo cardiomyopathy. Circulation 126, 697-706 (2012).

5. Y-Hassan, S. \& Henareh, L. Plasma catecholamine levels in patients with Takotsubo syndrome: implications for the pathogenesis of the disease. Int. J. Cardiol. 181, 35-38 (2015).

6. Samuels, M. A. The brain-heart connection. Circulation 116, 77-84 (2007).

7. Madias, J. E. Low prevalence of diabetes mellitus in patients with Takotsubo syndrome: a plausible 'protective' effect with pathophysiologic connotations. Eur. Heart J. Acute Cardiovasc. Care http://dx.doi.org/ 10.1177/2048872615570761.

8. Abroug, F. et al. Scorpion-related cardiomyopathy: clinical characteristics, pathophysiology, and treatment. Clin. Toxicol. (Phila.) 8, 1-8 (2015).

9. Madias, J. E. Appropriate implementation of echocardiography in Takotsubo syndrome: earlier and more frequently. Echocardiography 30, 1123-1125 (2013).

10. Spencer, K. T. et al. Focused cardiac ultrasound: recommendations from the American Society of Echocardiography. J. Am. Soc. Echocardiogr. 26, 567-581 (2013).

11. Madias, J. E. A proposal for a pathogenesisseeking animal model of Takotsubo syndrome. Am. J. Cardiol. 111, 1231-1232 (2013).

12. Redfors, B., Shao, Y., Ali, A. \& Omerovic, E. Are the different patterns of stress-induced (Takotsubo) cardiomyopathy explained by regional mechanical overload and demand supply mismatch in selected ventricular regions? Med. Hypotheses 81, 954-960 (2013). 